\title{
Efeito da densidade de corrente e pH na obtenção da liga Ni-Fe por eletrodeposição
}

\author{
Effect of current density and $\mathrm{pH}$ in obtaining the \\ $\mathrm{Ni}-\mathrm{Fe}$ alloy by electrodeposition
}

José Anderson Machado Oliveira ${ }^{1}$, Anamélia de Medeiros Dantas Raulino ${ }^{1}$,
José Leonardo Costa Raulino ${ }^{1}$, Ana Regina Nascimento Campos ${ }^{1}$,
Shiva Prasad ${ }^{1}$, Renato Alexandre Costa de Santana

\begin{abstract}
${ }^{1}$ Laboratório de Eletroquímica e Corrosão - Universidade Federal de Campina Grande, Centro de Educação e Saúde, Sítio Olho D’água da Bica, S/N, CP: 58175-000, Cuité, PB, Brasil.

e-mail: jmo.anderson@gmail.com, anameliadantas@yahoo.com.br, leonardo.jcr@hotmail.com, arncampos@yahoo.com.br, prasad@deq.ufcg.edu.br, renatoacs@ufcg.edu.br
\end{abstract}

\section{RESUMO}

Revestimentos metálicos podem ser aplicados para diferentes finalidades, como exemplo, para melhorar as propriedades mecânicas, catalíticas, anticorrosivas ou simplesmente melhorar o aspecto decorativo. Neste trabalho foram obtidas ligas de Ni-Fe pelo processo de eletrodeposição utilizando um banho eletrolítico simples contendo os seguintes reagentes, sulfato de níquel, sulfato de ferro e tartarato de sódio. Como ferramenta de otimização foi utilizado um planejamento experimental completo $2^{2}$, associado à técnica de metodologia de superfície de resposta (MSR). Foram feitas medidas de composição química, eficiência de corrente, morfologia superficial e medidas eletroquímicas de corrosão. Foi observado que a diminuição do $\mathrm{pH}$ favoreceu o aumento do ferro e diminuição do níquel na liga. O teor de ferro influenciou na morfologia da liga. O experimento ótimo apresentou uma resistência à corrosão média de $5471,5 \Omega . \mathrm{cm}^{2}$ e uma densidade de corrente de corrosão média de $4,814 \times 10^{-6} \mathrm{~A} / \mathrm{cm}^{2}$, este experimento apresentou uma composição percentual em massa de níquel de $70 \mathrm{wt} \%$ e de ferro de $30 \mathrm{wt} \%$ e uma eficiência de corrente média de $58,7 \%$.

Palavras-chave: Liga Ni-Fe, Eletrodeposição, Corrosão, Planejamento Experimental.

\begin{abstract}
Metallic coatings can be applied for different purposes, for example to improve the mechanical, catalytic, anticorrosive properties or simply to improve the decorative appearance. In the work the Fe-Ni alloys have been obtained by electrodeposition process using a simple electrolytic bath containing the reagents, nickel sulfate, iron sulfate and sodium tartrate. A complete experimental design $2^{2}$, associated with the response surface methodology (RSM) technique was used as optimization tool. Chemical composition, current efficiency, surface morphology and electrochemical corrosion measures were performed. It was observed that a decrease in the $\mathrm{pH}$ favored an increase in iron and decrease in nickel contents in the alloy. The iron content influenced the alloy morphology. The best experiment showed an average corrosion resistance $5471.5 \Omega . \mathrm{cm}^{2}$ and a corrosion current density $4.814 \times 10^{-6} \mathrm{~A} / \mathrm{cm}^{2}$. This experiment presented a composition of $70 \mathrm{wt} \% \mathrm{Ni}$ and $30 \mathrm{wt} \% \mathrm{Fe}$ in the alloy and an average deposition current efficiency of $58.7 \%$.
\end{abstract}

Keywords: Ni-Fe alloy, Electrodeposion, Corrosion, Experimental Design.

\section{INTRODUÇÃO}

Revestimentos formados por ligas Ni-Fe apresentam excelentes propriedades, como, por exemplo, baixa coercividade, alta permeabilidade magnética e elevada resistência à tensão, podendo ser aplicadas em diversos setores industriais [1-3]. Estas ligas são utilizadas na construção de dispositivos eletrônicos, em revestimentos protetores e decorativos. Desta forma, nota-se a importância do desenvolvimento da liga de Ni-Fe para ampliar suas aplicações industriais [4,5]. 
Diferentes técnicas podem ser utilizadas para obtenção de revestimentos formados por ligas de Ni-Fe, por exemplo, pulverização catódica, fusão a laser, eletrodeposição, entre outras [6]. Entre elas, destaca-se a eletrodeposição que permite a obtenção de revestimentos metálicos finos na forma de metais puros ou ligas, sob controle dos parâmetros operacionais do sistema. O processo de eletrodeposição permite a obtenção de revestimentos sobre diversos tipos de materiais, até mesmo aqueles com geometrias complexas, estes podem ser caracterizados quanto a sua composição química, morfologia da superfície, resistência à corrosão, microdureza, entre outros. Desta forma, a eletrodeposição torna-se uma alternativa viável quando comparada a outras técnicas utilizadas para obtenção de revestimentos de metais puros ou ligas $[6,7]$

Estudos relatam que no processo de formação da liga de Ni-Fe obtida por eletrodeposição que o ferro deposita preferencialmente com relação ao níquel, esse fenômeno é conhecido como codeposição anômala [8] Na codeposição anômala ocorre a inibição da redução do níquel devido à precipitação ou adsorção do hidróxido de ferro II, $\mathrm{Fe}(\mathrm{OH})_{2}$, na superfície do revestimento $[9,10]$. Essa inibição é influenciada pelos parâmetros de deposição como a densidade de corrente e o pH do banho eletrolítico, fazendo necessário um maior número de investigações para contribuir com informações sobre a liga Ni-Fe [11-13].

Nos últimos anos o uso do planejamento experimental vem sendo utilizado em trabalhos na área de eletroquímica como ferramenta de otimização [14-16]. Sua utilização associado a técnicas como a metodologia de superfície de resposta (MSR) pode auxiliar no estudo dos parâmetros de obtenção das ligas metálicas com composição e propriedades características para cada tipo de aplicação. A vantagem da utilização de planejamentos fatoriais baseia-se na possibilidade de avaliação simultânea mais das variáveis e, dessa forma, definir as melhores condições experimentais para obtenção dos melhores revestimentos, além de diminuir a quantidade de experimentos e os custos $[8,16,17]$.

Neste trabalho foram obtidas ligas de Ni-Fe por eletrodeposição utilizado um banho eletrolítico contendo como agente complexante o tartarato de sódio, diferente de outros trabalhos que utilizaram como complexante o citrato de sódio ou ácido bórico. Foi avaliado a influência da densidade de corrente e do pH na composição química, morfologia e resistência a corrosão dos revestimentos obtidos. Como ferramenta de otimização foi utilizado o planejamento experimental completo associado à metodologia de superfície de resposta (MSR).

\section{MATERIAIS E MÉTODOS}

Na preparação do banho eletrolítico para eletrodeposição da liga foram utilizados reagentes de grau analítico dissolvidos em água destilada e deionizada. $\mathrm{O}$ pH da solução foi ajustado com solução de $\mathrm{H}_{2} \mathrm{SO}_{4}(50 \% \mathrm{v} / \mathrm{v})$ ou $\mathrm{NH}_{4} \mathrm{OH}$ (concentrado). A composição química do eletrólito é apresentada na Tabela 1. Foram utilizados três reagentes na preparação do banho, sulfato de níquel, sulfato de ferro e tartarato de sódio, diferentemente da maioria dos trabalhos reportados na literatura que utilizaram banhos mais complexos $[5,11,18,19]$.

Tabela 1: Composição química do banho eletrolítico utilizado para eletrodeposição da liga Ni-Fe.

\begin{tabular}{c|c|c}
\hline Reagentes & Concentração $\left(\mathbf{m o l}^{-L^{-1}}\right)$ & Função \\
\hline $\mathrm{NiSO}_{4} \cdot 6 \mathrm{H}_{2} \mathrm{O}$ & 0,30 & Fonte de níquel \\
\hline $\mathrm{FeSO}_{4} \cdot 7 \mathrm{H}_{2} \mathrm{O}$ & 0,06 & Fonte de ferro \\
\hline $\mathrm{C}_{4} \mathrm{H}_{4} \mathrm{Na}_{2} \mathrm{O}_{6} \cdot 2 \mathrm{H}_{2} \mathrm{O}$ & 0,40 & Complexante \\
\hline
\end{tabular}

A eletrodeposição foi realizada sobre chapas planas de cobre com área de $8 \mathrm{~cm}^{2}$ (eletrodo de trabalho). Antes do início do processo o eletrodo de trabalho foi polido mecanicamente com lixas de carbeto de silício em granulação decrescente de 400, 600 e 1200. Seguido de imersão em soluções de $\mathrm{NaOH}(10 \% \mathrm{~m} / \mathrm{v})$ para desengraxe, e em solução de $\mathrm{H}_{2} \mathrm{SO}_{4}(1 \% \mathrm{v} / \mathrm{v})$ para ativação da superfície do eletrodo.

A eletrodeposição foi conduzida em controle galvanostático utilizando uma fonte de energia da MINIPA modelo MPL-1303M, em temperatura ambiente de $25 \pm 2{ }^{\circ} \mathrm{C}$. Como eletrodo auxiliar foi utilizado uma malha cilíndrica oca de platina. Em todos os experimentos foi utilizada uma carga de 300 Coulombs. O tempo utilizado na eletrodeposição foi calculado de acordo com a carga total aplicada e a densidade de corrente do processo. A Equação 1 foi utilizada no cálculo da eficiência de corrente (EC) do processo de eletrodeposição.

$\mathrm{EC}=\frac{w}{E w * I * \frac{t}{F}}=\frac{w * F}{I * t} \sum \frac{c i * n i}{M i} * 100$ 
Onde $w$ é a massa do depósito (g), $t$ o tempo utilizado na deposição (s), $I$ a corrente total utilizada no processo (A), Ew o peso equivalente da liga obtida (gramas por equivalente), $c i$ a fração em peso do elemento no depósito, $n i$ o número de elétrons transferidos por cada átomo de metal, $M i$ a massa atômica do elemento (g.mol $\left.{ }^{-1}\right)$ e $F$ a constante de Faraday $\left(96,485\right.$ C.mol $\left.{ }^{-1}\right)[8,20,21]$.

Os experimentos foram conduzidos utilizando um planejamento fatorial completo $2^{2}$, mais dois pontos centrais. Para avaliação e otimização do processo foi utilizada a técnica de metodologia de superfície de resposta (MSR). As variáveis foram avaliadas nos níveis codificados baixo $(-1)$, central $(0)$ e alto $(+1)$. Os experimentos foram realizados em duplicata e em ordem aleatória para evitar erros sistemáticos. Para análise estatística dos resultados foi utilizado o pacote estatístico do software STATISTICAC versão 8.0 [8,16,22]. A Tabela 2 apresenta os valores reais e codificados dos parâmetros operacionais avaliados neste trabalho.

Tabela 2: Níveis reais e codificados das variáveis do planejamento fatorial $2^{2}$.

\begin{tabular}{c|c|c|c}
\hline Variáveis & Nível -1 & Nível 0 & Nível +1 \\
\hline Densidade de corrente $\left(\mathrm{mA} . \mathrm{cm}^{-2}\right)$ & 20 & 60 & 100 \\
\hline $\mathrm{pH}$ & 3 & 4,5 & 6 \\
\hline
\end{tabular}

A composição química dos revestimentos foi obtida pela técnica de energia dispersiva de raios-X (EDX) utilizando um espectrômetro de fluorescência de raios-X por energia dispersiva. A morfologia dos revestimentos foi avaliada pela técnica de Microscopia Eletrônica de Varredura (MEV) utilizando um microscópio eletrônico da TESCAN modelo VEGA 3SBH com Detector de EDS a seco da Oxford modelo XACT IE150.

Para os ensaios eletroquímicos de corrosão foram utilizadas as técnicas de Polarização Potenciodinâmica Linear (PPL) e Espectroscopia de Impedância Eletroquímica (EIE), utilizando um potenciostato/galvanostato PG STATE 30 da AUTOLAB. Para obtenção dos dados de PPL e EIE foram utilizados os softwares GPES e FRA2, respectivamente. Os ensaios foram realizados em uma célula composta pelo eletrodo de trabalho revestido com a liga obtida, apresentando uma área exposta para análise de $0,76 \mathrm{~cm}^{2}, \mathrm{um}$ eletrodo auxiliar de platina em formato de espiral e um eletrodo de calomelano saturado (ECS) como eletrodo de referência. A velocidade de varredura para obtenção das curvas de PPL foi de $1 \mathrm{mV} \cdot \mathrm{s}^{-1}$. Antes dos ensaios de PPL e EIE foi utilizado um tempo de estabilização de 1200 segundos. Os ensaios de EIE foram realizados com um intervalo de frequência na ordem de $10 \mathrm{kHz}$ a $0,004 \mathrm{~Hz}$ com uma amplitude de $0,01 \mathrm{~V}$ no potencial de circuito aberto $\left(\mathrm{E}_{\mathrm{PCA}}\right)$. Os revestimentos selecionados dos ensaios de polarização, sendo eles, o melhor e o pior revestimento para o resultado de resistência à corrosão foram submetidos a ensaios de imersão em solução salina $(\mathrm{NaCl})$ em diferentes tempos: $0 \mathrm{~h}, 1 \mathrm{~h}, 24 \mathrm{~h}$ e $120 \mathrm{~h}$ utilizando a técnica de EIE. Os testes de corrosão foram realizados em solução de $\mathrm{NaCl}(3,5 \% \mathrm{~m} / \mathrm{v})$ a temperatura ambiente de $25 \pm 2{ }^{\circ} \mathrm{C}$. Os valores da resistência de polarização (Rp) foram calculados utilizando a equação de Stern-Geary (Equações 2 e 3) [23].

$$
\mathrm{Rp}=\frac{B}{\text { jcorr }}
$$

Nesta equação $R_{p}$ está em $\Omega . \mathrm{cm}^{2}, \mathrm{j}_{\text {corr }}$ em $\mu \mathrm{A} / \mathrm{cm}^{2}$ e B em V. O coeficiente de Stern-Geary (B) pode ser determinado, utilizando os coeficientes de Tafel, da seguinte maneira:

$$
B=\frac{\beta a \cdot \beta c}{2,303(\beta a+\beta c)}
$$

Onde $\beta \mathrm{a}$ e $\beta$ c são os coeficientes anódico e catódico, respectivamente, das retas de Tafel. A espessura dos revestimentos foi calculada utilizando a Equação 4.

$\mathrm{L}=\mathrm{P} / \mathrm{ad}$

Onde L é a espessura calculada, $\mathrm{P}$ é o peso do depósito, $a$ é a área depositada e $d$ a densidade do material depositado. O peso do depósito pode ser obtido utilizando a Equação 5.

$\mathrm{P}=(\mathrm{It} / \mathrm{F})^{*}(\mathrm{~A} / \mathrm{n})$ 
Onde P é o peso do depósito, I a corrente utilizada na deposição, t é o tempo do processo de deposição, F é a constante de Faraday, A é a área do eletrodo e n é o número de elétrons envolvidos na reação.

\section{RESULTADOS E DISCUSSÃO}

Foram obtidos com sucesso revestimentos de níquel e ferro pelo processo de eletrodeposição. Os mesmos mostraram ser brilhantes e aderentes ao substrato. Os resultados de composição química comprovam a deposição da liga Ni-Fe sobre o substrato de cobre. A Tabela 3 mostra a matriz do planejamento experimental com os níveis reais e codificados, os resultados de composição química em percentual em massa (wt\%), espessura dos revestimentos $(\mu \mathrm{m})$ e a eficiência de corrente (EC) em porcentagem (\%) da liga Ni-Fe.

Tabela 3: Matriz de planejamento experimental com os níveis reais e codificados, os resultados de composição química e eficiência de corrente do processo de eletrodeposição da liga Ni-Fe.

\begin{tabular}{c|c|c|c|c|c|c}
\hline Exp. & pH & Densidade $(\mathbf{m A . c m})^{-2}$ & Ni (wt\%) & Fe (wt\%) & Espessura $(\boldsymbol{\mu m})$ & EC (\%) \\
\hline 1 & $-1(3)$ & $-1(20)$ & 23 & 77 & 1,82 & 30 \\
\hline 2 & $-1(3)$ & $+1(100)$ & 39 & 61 & 2,87 & 37 \\
\hline 3 & $+1(6)$ & $-1(20)$ & 27 & 73 & 4,52 & 52 \\
\hline 4 & $+1(6)$ & $+1(100)$ & 79 & 21 & 3,21 & 44 \\
\hline $5(\mathrm{PC})$ & $0(4,5)$ & $0(60)$ & 70 & 30 & 3,88 & 58 \\
\hline $6(\mathrm{PC})$ & $0(4,5)$ & $0(60)$ & 70 & 30 & 3,96 & 59 \\
\hline
\end{tabular}

A Figura 1 apresenta a superfície de contorno, obtida pela técnica de metodologia de superfície de resposta (MSR), para composição percentual em massa de níquel no revestimento.

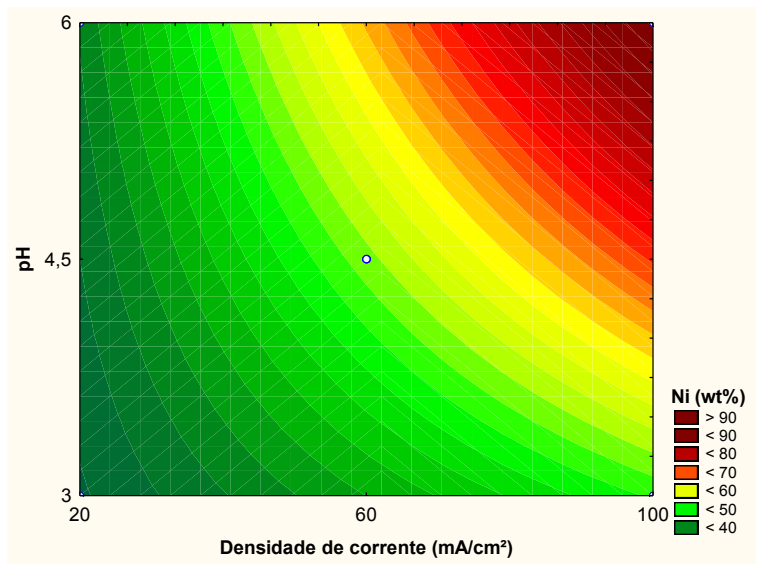

Figura 1: Superfície de contorno do percentual em massa de níquel (wt\%) na liga Ni-Fe em função das variáveis: $\mathrm{pH}$ e densidade de corrente.

Foi avaliado o efeito da densidade de corrente no processo de eletrodeposição da liga Ni-Fe. Observou-se que a composição percentual em massa de níquel foi favorecida com ou aumento da densidade de corrente, isto é, à medida que aumenta a densidade de corrente aumenta o percentual de níquel no revestimento. A Figura 2 mostra que a diminuição da densidade de corrente favorece o aumento percentual de ferro no revestimento. Neste trabalho foi utilizado como complexante o tartarato de sódio e foi observado o mesmo comportamento encontrado para os revestimentos obtidos com ácido bórico [10, 24]. Este comportamento pode estar associado à limitação no transporte de massa de íons $\mathrm{Fe}^{2+}$ até a superfície do cátodo com o aumento da densidade de corrente, sendo assim, fator limitante do processo redução do ferro[10, 24]. Comportamento semelhante foi encontrado por Robotin et al. [24] em seus estudos sobre a liga Ni-Fe obtida com e sem a presença do ácido bórico no banho eletrolítico. 


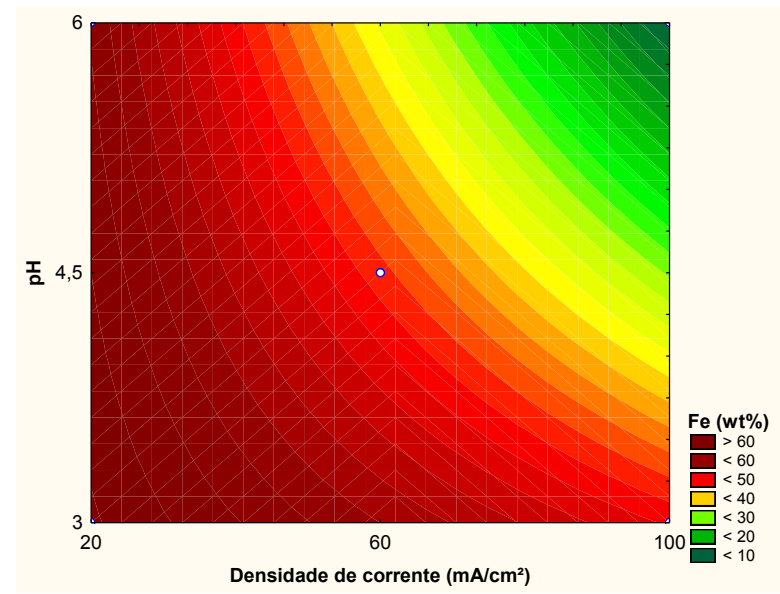

Figura 2: Superfície de contorno do percentual em massa de ferro (wt\%) na liga Ni-Fe em função das variáveis: $\mathrm{pH}$ e densidade de corrente.

Além da densidade de corrente foi avaliado a influência do $\mathrm{pH}$ na composição química dos revestimentos. $\mathrm{O} \mathrm{pH}$ foi avaliado de 3 a 6 e foi observado que com o aumento do $\mathrm{pH}$ foram obtidos revestimentos ricos em níquel e consequentemente com a diminuição do $\mathrm{pH}$ foram obtidos revestimentos ricos em ferro. Este comportamento pode ser associado à modificação do mecanismo de redução e da estrutura do complexante com a variação do $\mathrm{pH}$. Segundo Hessami e Tobias [25] a redução da liga Ni-Fe é baseado na presença de espécies solúveis de $\mathrm{FeOH}^{+}$e $\mathrm{NiOH}^{+}$presentes no banho eletrolítico. Matlosz [26] em seus estudos confirmou que a redução do níquel e do ferro ocorre a partir dos íons $\mathrm{NiOH}^{+}$e $\mathrm{FeOH}^{+}$respectivamente. Com o aumento $\mathrm{pH}$ diminui a formação de $\mathrm{FeOH}^{+}$e aumenta de $\mathrm{NiOH}^{+}$favorecendo assim, o aumento do percentual de níquel no revestimento.

De acordo com a Tabela 3 a maior eficiência de corrente (EC) foi obtida com densidade de corrente de $60 \mathrm{~mA} \cdot \mathrm{cm}^{-2} \mathrm{e} \mathrm{pH} \mathrm{4,5} \mathrm{com} \mathrm{valor} \mathrm{aproximado} \mathrm{de} 58 \%$. Resultados de eficiência semelhantes foram observados na literatura para deposição da liga Ni-Fe [18,24]. Ligas ricas em níquel e com valores baixos de ferro favorecem o aumento da EC. A dependência da eficiência de corrente em relação à densidade de corrente aplicada ao processo de eletrodeposição pode ser explicada pela influência da reação de redução de hidrogênio que consumirá uma parcela da corrente aplicada, assim, valores elevados de densidade de corrente favorecem a diminuição da $\mathrm{EC}$. $\mathrm{O}$ aumento do $\mathrm{pH}$ favorece o aumento da $\mathrm{EC}$, pois quanto menor for o valor do $\mathrm{pH}$ do banho eletrolítico maior será o aumento da reação de evolução de hidrogênio e consequentemente maior será a diminuição da EC [24]

A técnica de microscopia eletrônica de varredura (MEV) foi utilizada para caracterização da morfologia superficial da liga Ni-Fe. A Figura 3 apresenta imagens da superfície do melhor (Exp. 5: pH 4,5 e $60 \mathrm{~mA} . \mathrm{cm}^{-2}$, nível 0) e do pior (Exp. 1: pH 3 e 20 mA.cm ${ }^{-2}$, níveis: -1 e -1) revestimento, com ampliações de 500 e 1000 X (vezes), selecionados de acordo com os resultados dos ensaios eletroquímicos de corrosão, isto é, melhores resultados de resistência à polarização (Tab. 4).
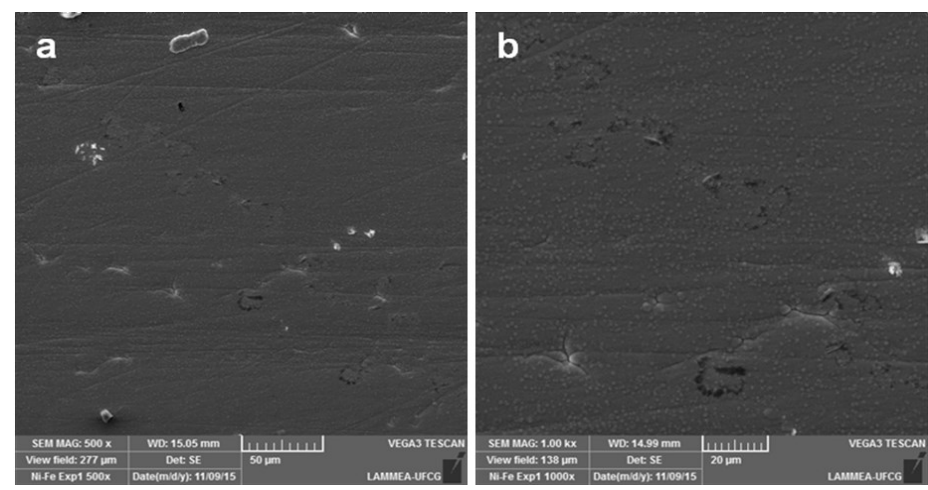
OLIVEIRA, J.A.M.; RAULINO, A. M. D.; RAULINO, J. L. C.; CAMPOS, A.R.N.; PRASAD, S.; SANTANA, R.A.C. revista Matéria, v.22, n.1, 2017.
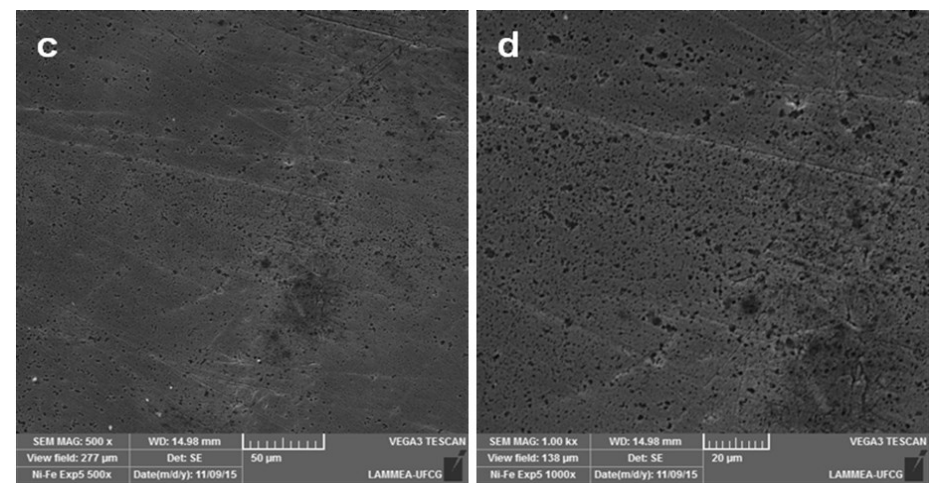

Figura 3: Micrografias (MEV) da superfície dos revestimentos da liga Ni-Fe: a) Exp. 1 (500 X); b) Exp. 1 $(1000 \mathrm{X})$; c) Exp. 5 (500 X); d) Exp. 5 (1000 X).

Observa-se que o revestimento recobriu toda superfície do eletrodo, tanto para o melhor experimento (exp. 5) quanto para o pior experimento (exp. 1). Observa-se, também, a possível formação de cristais não definidos e a presença de micronódulos no experimento 1 (pior). Com o aumento do teor de ferro na liga ocorre o refinamento dos grãos na superfície dos revestimentos. Este comportamento está relacionado com a diminuição do $\mathrm{pH}$ que favorece o aumento da teor de ferro na liga e consequentemente o refinamento dos grãos. As imagens de MEV para o experimento 5 (melhor) apresenta uma morfologia porosa e sem a presença de nódulos. Uma possível explicação para variações na morfologia superficial do revestimento pode estar relacionada com a mudança na orientação de crescimento na microestrutura do depósito, este fenômeno está diretamente relacionado com o aumento no controle por difusão em elevadas densidades de corrente no processo de eletrodeposição que aumenta a velocidade de crescimento dos cristais favorecendo a formação de películas com menor porosidade e homogêneas. Resultado de morfologia semelhante foi relatado na literatura para depósitos da liga Ni-Fe obtida em diferentes densidades de corrente e temperatura do banho eletrolítico [27].

Os ensaios eletroquímicos de corrosão foram realizados em meio de $\mathrm{NaCl}(3,5 \% \mathrm{~m} / \mathrm{v})$ a temperatura ambiente de $25 \pm 2{ }^{\circ} \mathrm{C}$. Os valores de resistência à polarização $(\mathrm{Rp})$ e densidade de corrente de corrosão $\left(\mathrm{j}_{\text {corr }}\right)$, extraídos das curvas de polarização (PPL), são apresentados na Tabela 4.

Tabela 4: Resultados das medidas de polarização potenciodinâmica linear (PPL) da liga Ni-Fe, feitas em meio de $\mathrm{NaCl}$ $(3,5 \% \mathrm{~m} / \mathrm{v})$ e temperatura de $25 \pm 2{ }^{\circ} \mathrm{C}$.

\begin{tabular}{|c|c|c|c|c|}
\hline Exp. & pH & Densidade $\left(\mathrm{mA} . \mathrm{cm}^{-2}\right)$ & $\operatorname{Rp}\left(\Omega . \mathrm{cm}^{2}\right)$ & $\mathrm{j}_{\text {corr }}\left(\mathrm{A} \cdot \mathrm{cm}^{-2}\right)$ \\
\hline 1 & $-1(3)$ & $-1(20)$ & 575,1 & $5,65 \times 10^{-5}$ \\
\hline 2 & $-1(3)$ & $+1(100)$ & 2505 & $1,598 \times 10^{-5}$ \\
\hline 3 & $+1(6)$ & $-1(20)$ & 1273 & $2,227 \times 10^{-5}$ \\
\hline 4 & $+1(6)$ & $+1(100)$ & 2650 & $1,928 \times 10^{-5}$ \\
\hline $5(\mathrm{PC})$ & $0(4,5)$ & $0(60)$ & 5143 & $5,212 \times 10^{-6}$ \\
\hline $6(\mathrm{PC})$ & $0(4,5)$ & $0(60)$ & 5800 & $4,417 \times 10^{-6}$ \\
\hline
\end{tabular}

Observa-se pelos resultados de corrosão que o experimento 1, obtido nas condições de pH 3 e densidade de corrente de $20 \mathrm{~mA} . \mathrm{cm}^{-2}$ (níveis -1 e -1), apresentou o menor valor de resistência à polarização $(575,1$ $\left.\Omega . \mathrm{cm}^{2}\right)$ e a maior densidade de corrente de corrosão $\left(5,65 \times 10^{-5} \mathrm{~A} . \mathrm{cm}^{-2}\right)$ sendo, portanto, considerado o pior experimento, ou seja, o que apresentou a menor resistência à corrosão. Já os melhores resultados foram observados nos experimentos realizados no ponto central do planejamento experimental, ou seja, pH 4,5 e densidade de corrente de $60 \mathrm{~mA} . \mathrm{cm}^{-2}$ (nível 0 ), obtendo uma resistência à polarização média de $5471,5 \Omega . \mathrm{cm}^{2} \mathrm{e}$

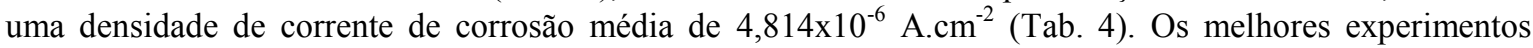
apresentaram uma maior proporção percentual em massa de níquel em relação ao pior experimento que apre- 
sentou uma maior proporção de ferro, assim, a presença do metal mais nobre em maior quantidade pode ser uma explicação para a maior resistência à corrosão apresentada por esses revestimentos. A maior resistência à corrosão pode estar associada também a morfologia do revestimento, pois o revestimento que apresentou maior resistência à polarização apresentou uma superfície porosa e uniforme sem aparecimento de trincas ou pontos que poderiam atuar como regiões preferenciais de corrosão. Já no experimento 1, que apresentou baixa resistência à corrosão, pode-se observar que a superfície do revestimento apresenta pontos que podem possibilitar o ataque ao substrato justificando o baixo valor de resistência à polarização. A Figura 5 apresenta as curvas de polarização do melhor e do pior revestimento, selecionados de acordo com os resultados eletroquímicos de corrosão (Tab. 4).

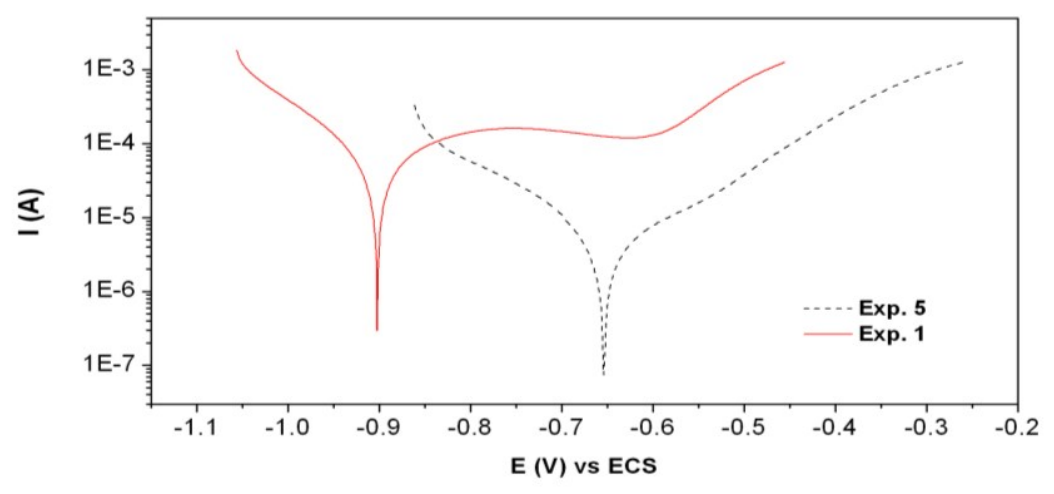

Figura 5: Curvas de polarização potenciodinâmica linear (PPL) da liga Ni-Fe, obtidas em meio de $\mathrm{NaCl}(3,5 \% \mathrm{~m} / \mathrm{v})$ e temperatura de $25 \pm 2{ }^{\circ} \mathrm{C}$.

Observa-se nas curvas PPL dos revestimentos selecionados um elevado deslocamento do potencial de corrosão para valores mais nobres no melhor experimento (o experimento 5 foi selecionado para representar o comportamento apresentado nos ensaios do ponto central do planejamento), em comparação ao experimento 1 (pior), sendo $-0,902 \mathrm{~V}$ para o pior (Exp. 1) e $-0,654 \mathrm{~V}$ para o melhor (Exp. 5). Observa-se, também, a possível formação de um filme de passivação na superfície do experimento 1, evidenciado pela diminuição da corrente no ramo anódico da curva de PPL em um intervalo de potencial entre $-0,8 \mathrm{~V}$ a $-0,65 \mathrm{~V}$, fato não observado para o experimento 5 .

Para confirma os resultados obtidos tanto nas curvas de PPL quanto nos diagramas de EIE foram realizados medidas de imersão dos revestimentos que obteve menor e maior resistência à polarização. Os revestimentos foram imersos em solução de $\mathrm{NaCl}$ durante 120 horas e foram realizadas medidas nos seguintes tempos: 0h, 1h, 24h e 120h. A Figura 6 mostra os diagramas de impedância de Nyquist para a liga Ni-Fe selecionada (Exp. 5), obtidas em potencial de circuito aberto ( $\left.\mathrm{E}_{\mathrm{PCA}}\right)$ após diferentes tempos de imersão. Foi observado que no tempo zero foi obtido o maior arco de impedância que pode ser associado ao processo de transferência de carga [28]. Depois de uma hora ocorreu uma diminuição de arco que mostra que ocorreu um fenômeno superficial, isto é, ocorreu dissolução do filme e provavelmente a formação de um filme de passivação instável. A formação desse filme de passivação pode ser confirmada pela não alteração no tamanho dos arcos capacitivos realizados nos tempos de 24 e 120 horas. Esse comportamento confirma que o experimento 5 possui a maior resistência à corrosão e pode estar associado a maior porosidade/rugosidade do revestimento que favoreceu a migração do níquel para superfície do revestimento favorecendo, assim, uma possível formação de um filme de passivação rico em níquel. 


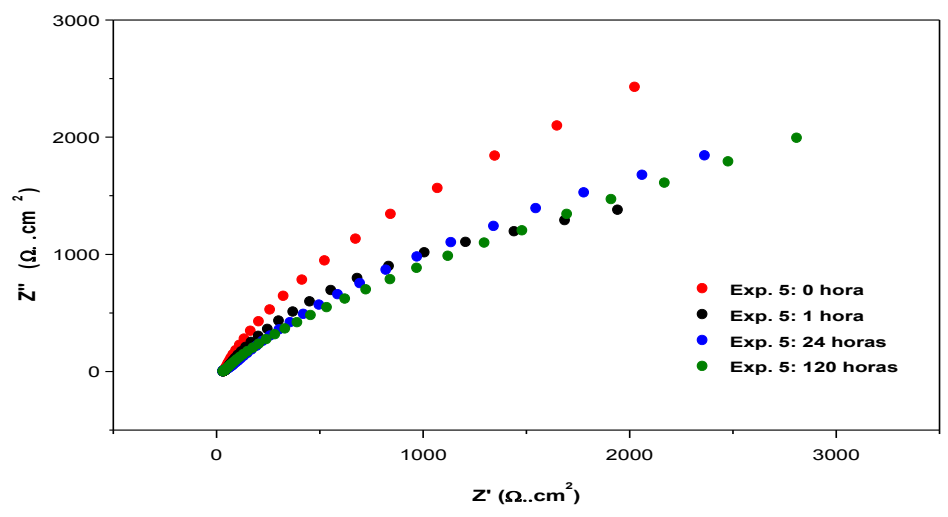

Figura 6: Diagramas de Nyquist (EIE) da liga Ni-Fe (Exp. 5), obtidos no $\mathrm{E}_{\mathrm{PCA}}$ em meio de $\mathrm{NaCl}(3,5 \% \mathrm{~m} / \mathrm{v})$ e temperatura de $25 \pm 2{ }^{\circ} \mathrm{C}$.

O teste de imersão também foi realizado para o experimento 1 (Fig. 7), que pelas análises de PPL e EIE foi o revestimento que apresentou menor resistência à corrosão. Para o experimento 1 não foi possível obter os Diagramas de Nyquist, para todos os tempos utilizados no ensaio do experimento 5, pois, em menos de 1 hora (uma hora) de imersão foi observado a dissolução do revestimento consequentemente diminuição da resistência à polarização. Após uma hora ocorreu o desprendimento do revestimento do substrato de cobre confirmando assim a dissolução do revestimento. Desta forma, fica evidente que esse revestimento apresentou uma baixa resistência à corrosão.

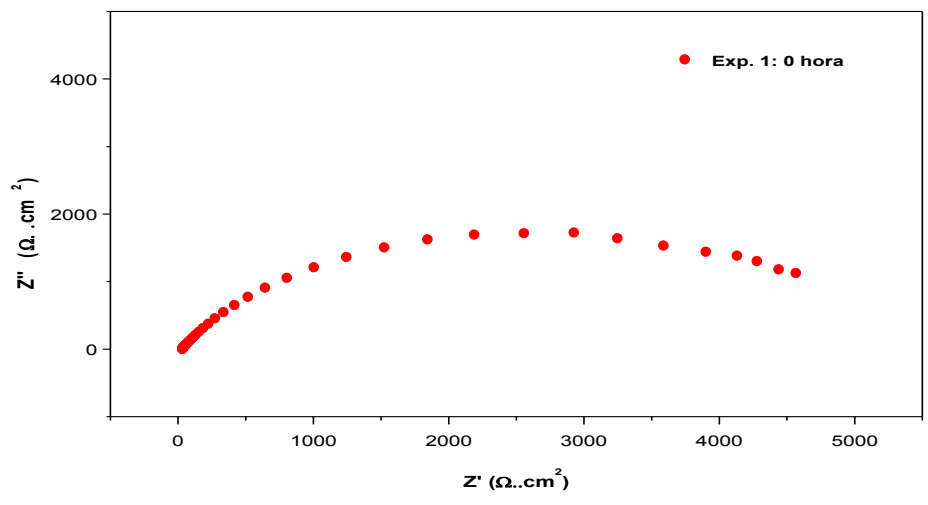

Figura 7: Diagramas de Nyquist (EIE) da liga Ni-Fe (Exp. 1), obtidos no $\mathrm{E}_{\mathrm{PCA}}$ em meio de $\mathrm{NaCl}(3,5 \% \mathrm{~m} / \mathrm{v})$ e temperatura de $25 \pm 2{ }^{\circ} \mathrm{C}$

\section{CONCLUSÕES}

A liga Ni-Fe foi obtida com sucesso utilizando um banho eletrolítico contendo como complexante o tartarato de sódio. Foi observado que o $\mathrm{pH}$ influenciou na composição química da liga. Com o aumento do valor do $\mathrm{pH}$ do banho ocorreu o aumento do teor de níquel no revestimento. Com a diminuição do pH o banho apresentou uma maior concentração de $\mathrm{FeOH}^{+}$favorecendo o aumento do teor de ferro na liga.

Os melhores resultados de resistência à corrosão foram observados nos experimentos realizados nos pontos centrais do planejamento experimental, apresentando uma resistência à polarização média de 5471,5 $\Omega . \mathrm{cm}^{-2}$ e uma densidade de corrente de corrosão média de $4,814 \times 10^{-6} \mathrm{~A} . \mathrm{cm}^{-2}$, estes experimentos apresentaram uma morfologia porosa e sem a presença de nódulos. Já o pior resultado de resistência à corrosão foi observado para o experimento 1 , obtido em pH 3 e $20 \mathrm{~mA} \cdot \mathrm{cm}^{-2}$, apresentando uma resistência à polarização de $575,1 \Omega . \mathrm{cm}^{-2}$ e uma densidade de corrente de corrosão de $5,65 \times 10^{-5} \mathrm{~A} . \mathrm{cm}^{-2}$, este experimento apresentou uma morfologia uniforme com a possível presença de cristais não bem definidos e de micronódulos na superfície do revestimento. Os testes de imersão comprovaram que o experimento que possui a maior porosidade 
OLIVEIRA, J.A.M.; RAULINO, A. M. D.; RAULINO, J. L. C.; CAMPOS, A.R.N.; PRASAD, S.; SANTANA, R.A.C. revista Matéria, v.22, n.1, 2017.

apresentou a maior resistência à corrosão pela possível formação de um filme de passivação na superfície do revestimento.

\section{AGRADECIMENTOS}

Os autores agradecem a Coordenação de Aperfeiçoamento de Pessoal de Nível Superior (CAPES), Conselho Nacional de Desenvolvimento Científico e Tecnológico (CNPq) e a Fundação de Apoio à Pesquisa do Estado da Paraíba (FAPESQ) pelo apoio financeiro, ao Laboratório de Eletroquímica e Corrosão e ao Laboratório de Microscopia Eletrônica do Departamento de Engenharia Mecânica da UFCG pelas análises de MEV e EDS.

\section{BIBLIOGRAFIA}

[1] LI, Z., SUN, X., ZHENG, Y., ZHANG, H. "Microstructure and magnetic properties of micro NiFe alloy arrays for MEMS application”, Journal of Micromechanics and Microengineering, v. 23, n. 8, pp. 085013, 2013.

[2] GIALLONARDO, J. D. "Structure and properties of electrodeposited nanocrystalline Ni and Ni-Fe alloy continuous foils", Thesis Doctorate Degree, University of Toronto, 2013

[3] CHANG, W., WEI, Y., GUO, J., et al. "Thermal stability of Ni-Fe alloy foils continuously electrodeposited in fluorborate bath”, Open Journal of Metal, n. 2, pp. 18-23, 2012.

[4] SHONGWE, M. B., DIOUF, S., DUROWOJU, M. O., et al. "Effect of sintering temperature on the microstructure and mechanical properties of $\mathrm{Fe}-30 \% \mathrm{Ni}$ alloys produced by spark plasma sintering", Journal of Alloys and Compounds, v. 649, pp. 824-832, 2015.

[5] MA, L., ZHANG, L., LI, X., et al. "Fabrication and characterization of electrodeposited nanocrystalline $\mathrm{Ni}-\mathrm{Fe}$ alloys for NiFe2O4 spinel coatings", Transactions of Nonferrous Metals Society of China, v. 25, n. 1, pp. 146-153, 2015.

[6] YU, Y. D., WEI, G. Y., GUO, H. F., et al. "Study on preparation of NiFeP films by pulse electrodeposition”, Surface Engineering, v. 28, n. 1, pp. 30-36, 2012.

[7] ARGAÑARAZ, M. P. Q., RIBOTTA, S. B., FOLQUER, M. E., et al., "The chemistry and structure of nickel-tungsten coatings obtained by pulse galvanostatic electrodeposition”, Electrochimica Acta, v. 72, pp. 87-93, 2012.

[8] OLIVEIRA, A. L. M., COSTA, J. D., SOUSA, M. B. et al., "Studies on electrodeposition and characterization of the Ni-W-Fe alloys coatings", Journal of Alloys and Compounds, v. 619, pp. 697-703, 2015.

[9] LIMA, T.G., ROCHA, B.C.C.A., BRAGA, A.V.C., et al. "Response surface modeling and voltammetric evaluation of Co-rich $\mathrm{Cu}-\mathrm{Co}$ alloy coatings obtained from glycine baths", Surface \& Coatings Technology, v. 276, pp. 606-617, 2015.

[10] NAKANO, H., MATSUNO, M., OUE, S., et al., "Mechanism of anomalous type electrodeposition of Fe-Ni alloy from sulfate solution”, Materials Transactions, v. 45, n. 11, pp. 3130-3135, 2004.

[11] LLAVONA, A., PEREZ, L., CARMEN SANCHEZ, M., et al., "Enhancement of anomalous codeposition in the synthesis of Fe-Ni alloys in nanopores”, Electrochimica Acta, v. 106, pp. 392-397, 2013.

[12] NEURÓHR, K., CSIK, A., VAD, K., et al., "Near-substrate composition depth profile of direct currentplated and pulse-plated Fe-Ni alloys", Electrochimica Acta, v. 103, n. 0, pp. 179-187, 2013.

[13] FRICOTEAUX, P., ROUSSE, C. "Influence of substrate, $\mathrm{pH}$ and magnetic field onto composition and current efficiency of electrodeposited Ni-Fe alloys", Journal of Electroanalytical Chemistry, v. 612, pp. 914, 2008.

[14] COSKUN, M.I., KARAHAN, I.H., YUCEL, Y. “Optimized electrodeposition concentrations for hydroxyapatite coatings on CoCrMo biomedical alloys by computational techniques", Electrochimica Acta, v. 150, pp 46-54, 2014.

[15] POROCH-SERITAN, M., CRETESCU, I., COJOCARU, C., et al., "Experimental design for modelling and multi-response optimization of Fe-Ni electroplating process", Chemical Engineering Research and Design, v. 96, pp. 138-149, 2015.

[16] SANTANA, R. A. C., CAMPOS, A. R. N., MEDEIROS, E. A., et al. "Studies on electrodeposition and corrosion behaviour of a Ni-W-Co amorphous alloy", Journal of Materials Science, v. 42, pp. 9137-9144, 2007. 
[17] SANTANA, R. A. C., PRASAD, S., MOURA, E. S., et al., "Studies on electrodeposition of corrosion resistant Ni-Fe-Mo alloy", Journal of Materials Science, v. 42, n. 7, pp. 2290-2296, 2007.

[18] MATSUI, I., MORI, H., KAWAKATSU, T., et al. "Enhancement in mechanical properties of bulk nanocrystalline $\mathrm{Fe}-\mathrm{Ni}$ alloys electrodeposited using propionic acid", Materials Science and Engineering: A, v. 607 , pp. 505-510, 2014.

[19] MATSUI, I., KAWAKATSU, T., TAKIGAWA, Y., et al. "Fabrication of bulk nanocrystalline Fe-Ni alloys with high strength and high ductility by an electrodeposition”, Materials Letters, v. 116, pp. 71-74, 2014.

[20] QIAO, X., LI, H., ZHAO, W., et al. "Effects of deposition temperature on electrodeposition of zincnickel alloy coatings", Electrochimica Acta, v. 89, pp. 771-777, 2013.

[21] HEGDE, A.C., VENKATAKRISHNA, K., ELIAZ, N. "Electrodeposition of Zn-Ni, Zn-Fe and Zn-NiFe alloys", Surface \& Coatings Technology, v. 205, pp. 2031-2041, 2010.

[22] SANTANA, R. A. C., CAMPOS, A. R. N., PRASAD, S. "Otimização do banho eletrolítico da liga Fe W-B resistente à corrosão", Quimica Nova, v. 30, n. 2, pp. 360-365, 2007.

[23] ALJOHANI, T. A., HAYDEN, B. E. "A simultaneous screening of the corrosion resistance of Ni-W thin film alloys", Electrochimica Acta, v. 111, pp. 930-936, 2013

[24] ROBOTIN, B., ISPAS, A., COMAN, V., et al. "Nickel recovery from electronic waste II Electrodeposition of Ni and Ni-Fe alloys from diluted sulfate solutions", Waste Management, v. 33, n. 11, pp. 2381-2389, 2013.

[25] HESSAMI, S., TOBIAS, C.W. "A Mathematical Model for Anomalous Codeposition of Nickel-Iron on a Rotating Disk Electrode”, Journal of the Electrochemical Society, v. 136, pp. 3611-3616, 1989.

[26] MATLOSZ, M. "Competitive Adsorption Effects in the Electrodeposition of Iron-Nickel Alloys", Journal of the Electrochemical Society, v 140, pp. 2272-2279, 1993.

[27] GAO, Y., WEI, G. Y., GE, H. L., et al. "Study on preparation of NiFe films by galvanostatic electrodeposition", Surface Engineering, v. 30, n. 2, pp. 97-101, 2014.

[28] RIBEIRO, D. V., SOUZA, S. A. C., ABRANTES, J. C. C. "Uso da Espectroscopia de Impedância Eletroquímica (EIE) para monitoramento da corrosão em concreto armado", Revista IBRACON de Estruturas e Materiais, v. 8, n. 4, pp. 529-546, 2015. 